\title{
Development of an automated method for in situ measurement of the geometrical properties of the ITER bolometer diagnostic
}

\author{
H. Meister, F. Penzel, L. Giannone, M. Kannamüller, A. Kling, J. Koll, T. Trautmann \\ Max-Planck-Institut für Plasmaphysik, EURATOM Association, Garching, Germany
}

\begin{abstract}
In order to derive the local emission profile of the plasma radiation in a fusion device using the line-integrated measurements of the bolometer diagnostic, tomographic reconstruction methods have to be applied to the measurements from many lines-of-sight. A successful reconstruction needs to take the finite sizes of detectors and apertures and the resulting non-ideal measurements into account. In ITER a method for in situ measurement of the geometrical properties of the various components of the bolometer diagnostic after installation is required as the viewing cones have to pass through narrow gaps between components. The method proposed to be used for ITER uses the beam of a laser with high intensity to illuminate the bolometer assembly from many different angles $\xi$ and $\theta$. A light-weight robot from Kuka Robotics is used to efficiently position the laser on many points covering the complete viewing cone of each line-of-sight and to direct the beam precisely into the entrance aperture of the bolometer. Measuring the response of the bolometer allows for the calculation of the transmission function $t(\xi, \theta)$, the angular etendue and finally the geometric function in reconstruction space, which is required for the tomography algorithms. Measuring the transmission function for a laboratory assembly demonstrates the viability of the proposed method. Results for a collimator-type camera from a prototype envisaged for ITER are presented. The implemented procedure is discussed in detail, in particular with respect to the automatisation applied which takes the achievable positioning and alignment accuracies of the robot into account. This discussion is extended towards the definition of requirements for a remote-handling tool for ITER.
\end{abstract}

Keywords: ITER, bolometer, geometric function, plasma diagnostics, measurement by laser beam, robot

\section{Introduction}

The total radiated power as well as the radiation emission profile on ITER will be determined by the bolometer diagnostic. A bolometer measures the plasma radiation over a wide spectral range (from soft- $X$ to the infrared) by monitoring the temperature rise induced by deposition of photon energy in the absorber layer of the bolometer. The reference detector type chosen for ITER is the metal resistor bolometer $[1,2]$. In order to derive the spatially resolved radiation emission profile from the line integrated measurements tomographic reconstruction methods are applied to the measurements of many lines-of-sight (e.g. [3] and references therein). However, the number of the lines-of-sight feasible in ITER is restricted due to the maximum amount of space available for diagnostic components and electrical feedthroughs. Thus, a successful reconstruction needs to take the finite sizes of detectors and apertures and the resulting non-ideal measurements into account [4]. In
ITER, a method for in situ measurement of the geometrical properties of the various components of the bolometer diagnostic after installation is required as the viewing cones have to pass through narrow gaps between components (e.g. for shielding) and small production tolerances might result in large deviations from the planned line-of-sight geometry due to the large size of ITER.

The method proposed to be used in ITER and its implementation in a laboratory environment are described in Section 2. First results from laboratory measurements and a comparison to the theoretically expected values are presented in Section 3. Finally, some requirements of such a method for the application in ITER will be derived in Section 4. 


\section{Experimental set-up}

The method proposed to be used for determining the geometrical properties of the ITER bolometer diagnostic components is based on previous work performed at JET [5]. The beam of a laser with high intensity is used to illuminate the bolometer assembly from many different angles $\xi$ (poloidal direction) and $\theta$ (toroidal direction). Measuring the response of the bolometer allows for the calculation of the transmission function $t(\xi, \theta)$, the angular etendue and finally the geometric function in reconstruction space, which is required for the tomography algorithms.

To efficiently transfer this method to an in situ measurement in ITER, one will have to make use of the remote-handling $(\mathrm{RH})$ equipment foreseen [6], e.g. the remote-handling arm. In order to asses the viability of using $\mathrm{RH}$-equipment and the requirements it has to meet, a light-weight robot from Kuka Robotics was procured and deployed in a laboratory environment. It was used to efficiently position the laser on many points covering the complete viewing cone of each line-of-sight and to direct the beam precisely into the entrance apertures of a collimator-type camera from a prototype envisaged for ITER.

Kuka's light-weight robot LBR4 features 7 rotational axes and has a positioning accuracy of $1 \mathrm{~mm}$ within a reach of $1178 \mathrm{~mm}$. The repeat accuracy is $0.05 \mathrm{~mm}$. The maximum payload of $14 \mathrm{~kg}$ is more than sufficient for our application. The robot provides a controller with a dedicated control and programming language. Amongst other features, several coordinate systems are implemented which can be chosen as the reference system for absolute or relative movements. For our measurements, the TOOL coordinate system has been defined such that the origin is at the entrance slit of the collimator and the axes coincide with the ones of the orientation of the laser and the robot. This allows for easy programming of angular movements around the entrance slit. The laser is positioned successively at angles $\xi$ and $\theta$ which cover the complete viewing cone of the collimator. At each position the laser is switched on and the deposited power on the bolometer detector is measured. Additionally, a research interface is provided to access the controller and its parameters via Ethernet connection from remote. This provides the possibility to control the movements of the robot via the data acquisition software of the bolometers and thus to precisely adjust the measurements with the movements. However, the final version was released only recently so that the full automatisation could not be implemented, yet.

The prototype bolometer camera used in the measure-

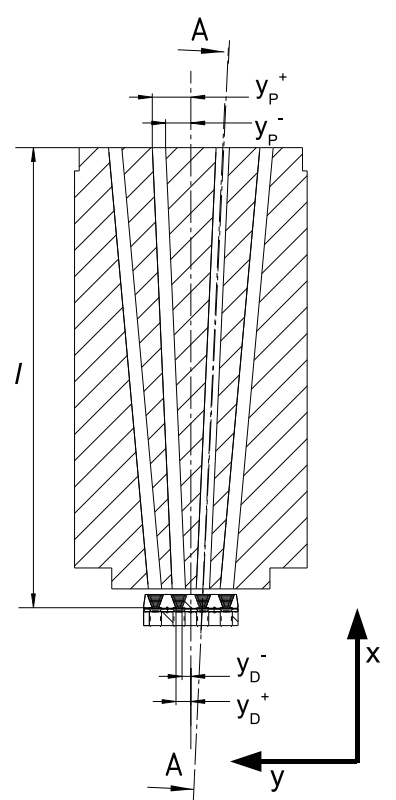

$A-A$
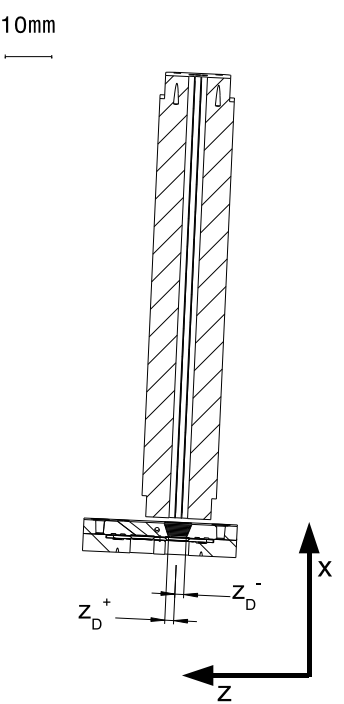

Figure 1: Sketch of the collimator prototype proposed for ITER and the coordinate system used. The sub-divisions of the collimator channels for restricting the viewing cone to narrower angles can be seen in the cut through a toroidal plane on the right hand side.

ments is sketched in figure 1. For compatibility with existing experiments and laboratory equipment this prototype features four channels whereas the final assembly for ITER is intended to have five. As bolometers in ITER will have to be placed behind shielding modules and have to view the plasma through narrow gaps (typically $20 \mathrm{~mm}$ wide and $500 \mathrm{~mm}$ long) between modules, the prototype collimator has additional sub-divisions in the toroidal direction in order to reduce the viewing cone lest that in-vessel components, which might move during operation, are not the limiting element. The coordinates and orientation chosen are given in figure 1 . The $x-y$ plane corresponds to the poloidal plane in ITER and the $x-z$ plane to the toroidal one.

A blue laser diode, emitting up to $10 \mathrm{~mW}$ at $405 \mathrm{~nm}$, is mounted on the tool flange of the robot and moved in the $x-y$ (angle $\xi$ ) and $x-z$ plane (angle $\theta$ ) using the robot's control features so that it always points into the entrance slit of one channel. The laser is defocussed to a spot size significantly larger than the entrance slit of the collimator to ensure constant power deposition. In order to enhance the accuracy of the robots orientation, position and orientation of robot and collimator have been measured using the FaroArm Platinum [7]. This tool can measure coordinates in three dimensions up to an accuracy of $0.016 \mathrm{~mm}$ within a radius of $1.8 \mathrm{~m}$. 


\section{Transmission function of the prototype collimator}

The theoretically expected transmission function $t_{i}(\xi, \theta)$ for each channel $i$ can be calculated analytically by considering the fraction of light from the laser reaching the detector:

$$
\begin{aligned}
I_{D, i}(\xi, \theta) & =I_{0} \frac{L_{\xi, i}(\xi) \cdot L_{\theta, i}(\theta)}{P} \cos \xi \cos \theta \\
& =I_{0} t_{\xi, i}(\xi) \cdot t_{\theta, i}(\theta)
\end{aligned}
$$

with the detected intensity $I_{D, i}$ on each channel $i$, the initial intensity of the laser $I_{0}$ and the area of the collimator entrance $P$. The illuminated area $A_{i}$ of each channel can be separated in its components $L_{\xi, i}(\xi)$ and $L_{\theta, i}(\theta)$ in $\xi$ and $\theta$ direction because the surfaces of detector and entrance slit are parallel. Neglecting reflections one gets in poloidal direction, similar to the results given in [5] for pin-hole cameras:

$$
t_{i}(\xi)= \begin{cases}0 & \text { for } \xi<\alpha_{1} \text { and } \xi>\alpha_{4} \\ \left(y_{D}^{+}-y_{P}^{-}+l \tan \xi\right) \cos \xi & \text { for } \alpha_{1}<\xi<\alpha_{2} \\ \left(y_{P}^{+}-y_{D}^{-}-l \tan \xi\right) \cos \xi & \text { for } \alpha_{3}<\xi<\alpha_{4}\end{cases}
$$

$y_{D, P}^{+,-}$denote the $y$-coordinates of lower resp. upper bound of the detector resp. the entrance slit of the collimator. $l$ gives the distance between the plane of entrance slit and detector (see figure 1). The angles $\alpha_{j}$ are defined by $\alpha_{1}=\arctan \left[\left(y_{P}^{-}-y_{D}^{+}\right) / l\right], \alpha_{2}=\arctan \left[\left(y_{P}^{-}-y_{D}^{-}\right) / l\right]$, $\alpha_{3}=\arctan \left[\left(y_{P}^{+}-y_{D}^{+}\right) / l\right], \alpha_{4}=\arctan \left[\left(y_{P}^{+}-y_{D}^{-}\right) / l\right]$.

Similar considerations lead to the transmission function in $\theta$ direction. However, the sub-divisions of the collimator (see fig. 1 have to be taken into account leading to more distinction of cases.

The transmission function has been measured as described in Section 2. The theoretically expected values have been calculated according to Eqs. (1) and (2) using the design data of the collimator. In the following, the measured transmission functions as well as the calculated ones are presented normalised to the respective maximum value, because the laser used was not absolutely calibrated. Fig. 2 shows the transmission in the (poloidal) $x-y$ plane for each of the four channels at the value of $\theta$ with maximum intensity. Measured values are denoted as symbols connected with dashed lines. The error bars in vertical direction give the standard deviation of the measured samples in steady state condition, the ones in horizontal direction are calculated using the positioning accuracy of the robot and its distance of $0.6 \mathrm{~m}$ from the collimator. Similarly, figure 3 compares the measured and calculated values in the (toroidal) $x-z$ plane, given at $\theta=1.5^{\circ}$, the angle with

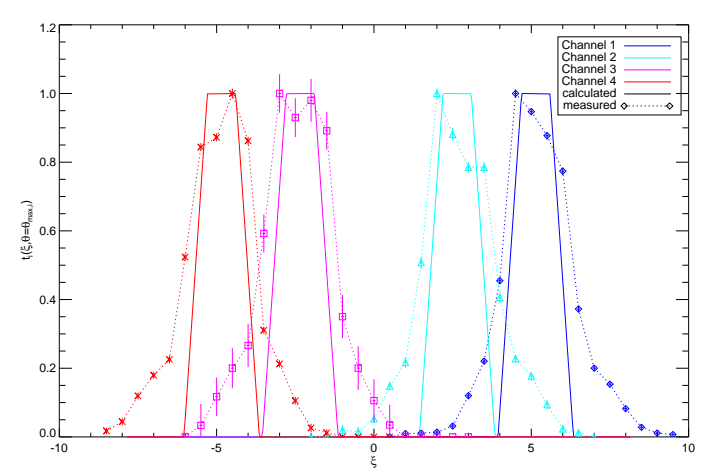

Figure 2: Measured (symbols with dashed lines) and calculated normalised transmission functions of the ITER prototype collimator in the (poloidal) $x-y$ plane given for each channel at the value of $\theta_{i}$ with maximum intensity.

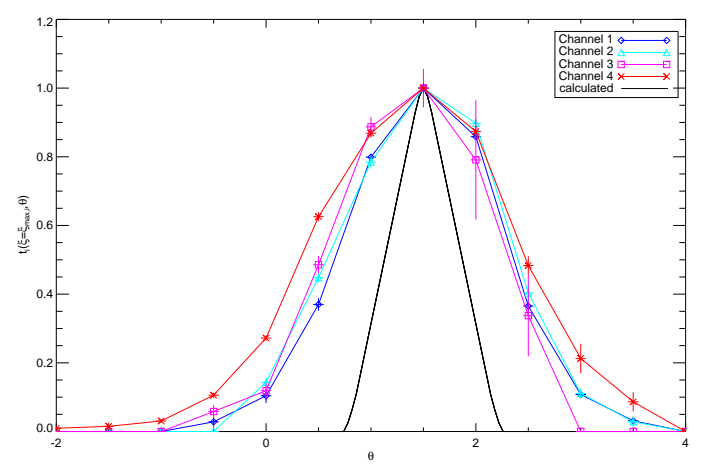

Figure 3: Measured (symbols) and calculated normalised transmission functions of the ITER prototype collimator in the (toroidal) $x-z$ plane given for each channel at the value of $\xi_{i}$ with maximum intensity. The $\theta$-coordinate of the calculated values had to be shifted by $1.5^{\circ}$.

highest intensity for all channels. Due to an erroneous alignment of the robot's orientation the values of the abscissae for $\theta$ had to be shifted by $1.5^{\circ}$ for the calculated transmission to match the measurements. However, as the shift is identical for all channels one can conclude that this method is well able to determine the exact orientation of a bolometer assembly.

The measured transmission curves are significantly broader than the calculated ones. It is assumed that this is due to reflections as no measures have been taken to reduce them and the collimator channels are manufactured by electro-erosion machining from the bulk which is made of TZM, an alloy of Ti, Zr and Mo featuring low thermal expansion and relatively high thermal conductivity. For the next measurements a coating of the 
collimator channels will be tried to investigate by how much the reflections can be reduced. Using threaded holes as proposed in [5] will not be feasible for ITER due to the much smaller dimensions of the collimator channels required.

Figure 4 gives a detailed overview of the measured and calculated transmission for channel 3. The contour levels are given in both cases at $10 \%$ intervals of the normalised values; the lowest contour is at $1 \%$ of the normalised value.

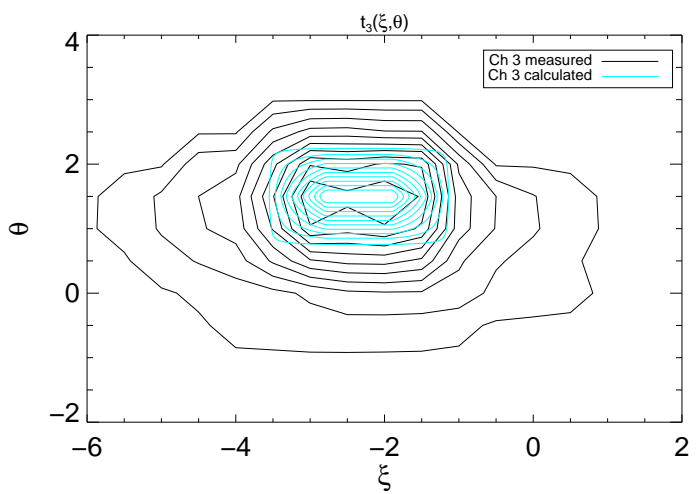

Figure 4: Measured (black) and calculated (light blue) transmission functions of channel 3 of the ITER prototype collimator. The lowest contour level is given at $1 \%$ the others at $10 \%$ intervals of the normalised intensity.

\section{Conclusions for the application in ITER}

The results shown in Section 3 are a proof of principle that the proposed method for an automatised in situ calibration of the ITER bolometer diagnostic is feasible. However, they do suggest several improvements and a number of conclusions can be drawn with respect to the requirements at ITER.

On the one hand it is clear that measures have to be devised to reduce the amount of stray light. Coatings like, e.g. $\mathrm{B}_{4} \mathrm{C}$, which also absorbs microwave radiation, have to be investigated. Also, the design, the manufacturing procedure and the tolerances given have to be optimised to define optimal reference points and surfaces for the exact measurement of position and orientation of the collimator. According to the manufacturer, the orientation of the collimator channels can be machined by electro-erosion up to an accuracy of $0.1^{\circ}$. This is the accuracy one at least has to achieve in the exact alignment of the robot.

The laser was moved in a radius of $0.6 \mathrm{~m}$ around the entrance slits of the collimator. Considering the positioning accuracy of the robot this relates to an angular accuracy of $0.1^{\circ}$. Taking the uncertainties of the measurements shown in figures 2 and 3 into account, this is sufficient. In ITER, the laser will have to be moved in a distance of about $2 \mathrm{~m}$ from the bolometer assemblies. Thus, the positioning accuracy of the RH-tool can be up to $3 \mathrm{~mm}$. However, the accuracy for pointing the laser on a spot in $2 \mathrm{~m}$ distance will have to be in the same order and its orientation will have to be known to within an accuracy of $0.1^{\circ}$.

Furthermore, some technical boundary conditions have to be mentioned, which proved to be important during the measurements. A RH-tool in ITER for in situ bolometer calibration will have to provide a flange for mounting the laser and some optics, which might sum up to a payload of $2 \mathrm{~kg}$. This payload needs to be moved precisely on prescribed coordinates. Additionally, electrical connections for the power supply and control of the laser have to be provided. For most lasers an RS232 interface will suffice. For good alignment and precise determination of current coordinates of the RH-tool a metrology systems with an accuracy of $0.5 \mathrm{~mm}$ or better needs to be in place. Together with appropriately defined procedures it will have to be used to determine the exact orientation of the RH-tool, too. The tests with the robot will have to be carried on to define viable procedures. Last but not least, the position control of the $\mathrm{RH}$-tool and the data acquisition of the bolometers have to be coordinated. Either the bolometer system triggers the movements of the RH-tool, as was implemented in the work presented, or the RH-tool triggers data acquisition, which might be the preferred choice of ITER. In both cases procedures need to be defined and implemented in close cooperation with the ITER CODAC and $\mathrm{RH}$ groups.

\section{Acknowledgments}

The authors wish to explicitly thank G. Fröhlich, S. Eder and W. Zeidner from the ASDEX Upgrade Team who performed the metrology measurements with the FaroArm.

This work was supported by funds from the German Ministry for Education and Research under the Grant No. 03FUS0006. The sole responsibility for the content presented lies with the authors.

\section{References}

[1] K. F. Mast, J. C. Vallet, C. Andelfinger, P. Betzler, H. Kraus, G. Schramm, A low noise highly integrated bolometer array for absolute measurement of vuv and soft x radaition, rsi 62 (1991) 744-750. 
[2] H. Meister, L. Giannone, L. D. Horton, G. Raupp, W. Zeidner, G. Grunda, S. Kalvin, U. Fischer, A. Serikov, S. Stickel, R. Reichle, The ITER bolometer diagnostic: status and plans, Review of Scientific Instruments 79 (2008).

[3] L. C. Ingesson, B. Alper, B. J. Peterson, J.-C. Vallet, Tomography diagnostics: Bolometry and soft-x-ray detection, in: A. E. Costley, D. W. Johnson (Eds.), Special issue on plasma diagnostics for magnetic fusion research, volume 53, Fusion Science and Technology, 2008.

[4] L. Ingesson, P. Bocker, R. Reichle, M. Romanelli, P. Smeulders, Projection-space methods to take into account finite beam width effects in two-dimensional tomography algorithms, Journal of the Optical Society of America A-Optics Image Science and Vision 16 (1999) 17-27.

[5] L. C. Ingesson, C. F. Maggi, R. Reichle, Characterization of geometrical detection-system properties for two-dimensional tomography, Review of Scientific Instruments 71 (2000) 1370-1378.

[6] I. Organisation, Design Description Document of WBS 2.3 Remote Handling, Technical Report, ITER Organisation, IDM: ITER_D_22KVFG, 1998.

[7] FARO EUROPE GmbH \& Co. KG, Product description FaroArm Platinum (2010) 